\title{
On the Accuracy of Denoising Algorithms in Medical Imaging: A Case Study
}

\author{
Fabrizio Russo \\ Department of Engineering and Architecture \\ University of Trieste \\ Trieste, Italy \\ rusfab@units.it
}

\begin{abstract}
Anisotropic diffusion-based filters are a widespread used resource for medical image denoising because they are designed to preserve the image details during noise removal. This paper aims at providing a quantitative evaluation of this important feature without the inaccuracies of the commonly adopted full-reference metrics. For the first time, the true value of detail preservation yielded by an anisotropic diffusion filter is formally derived from the filter theory. Many computer simulations are reported in the paper in order to study how values and locations of errors representing filtering distortion depend upon the parameter settings.
\end{abstract}

Keywords - image denoising, anisotropic diffusion, error analysis, detail preservation.

\section{INTRODUCTION}

The development of effective algorithms for noise reduction is a very active research area in medical imaging. Indeed, noise can significantly reduce the accuracy of operations such as feature extraction and object recognition that are of paramount importance for medical diagnosis. Unfortunately, data denoising is a very difficult task because fine details embedding diagnostic information can be destroyed during noise removal. In order to address this issue, many edge-preserving denoising algorithm have been proposed in the literature [1-2]. In this framework, some of most widely adopted methods are anisotropic diffusion filters [3-9], nonlocal means (NLM) [10-14] and wavelet-based approaches [1521]. Typical applications encompass (but are not limited to) ultrasound imaging [22-25], computed tomography (CT) [2627], magnetic resonance imaging (MRI) [28-32], data preprocessing for the extraction of biological information [3334], Since the accuracy of the filtering plays a key role, metrics that measure the filtering blur are necessary in order to analyze the actual performance of any denoising method and to validate it for medical purposes. Indeed, it is known that the peak signal-to-noise ratio (PSNR) cannot distinguish detail preservation from the overall noise cancellation and the same limitation also affects subjective approaches that aim at mimicking the human perception of image quality [35-36]. As shown in [37], these metrics can yield the same score for different mixtures of unprocessed noise and detail blur. The peak signal-to-blur ratio (PSBR) is a recently introduced full- reference measure that overcomes the drawbacks of the sole PSNR and other metrics in assessing the performance of grayscale image denoising filters [38]. Since the PSBR is the PSNR component that deals with the error due to filtering distortion, it can be adopted in conjunction with the classical and widespread adopted PSNR in order to fully characterize the behavior of a denoising system. Clearly, the method for blur estimation plays a key role in the accuracy of this method. The PSBR implementation adopting the most advanced algorithm for blur estimation [38] is not affected by apparent drawbacks, as typically occurs for other methods. Furthermore, it is able to yield the best results in all the comparisons with existing techniques for the measure of detail-preservation, such as vector metrics [39-41]. However, a critical issue with methods that perform separate estimates of residual noise and detail preservation consists in assessing the accuracy of such estimates. In this paper we show how a solution to this problem can be found for an important family of filters that are widely adopted for medical image denoising. Under the hypothesis of additive noise, we shall theoretically evaluate the PSBR for an anisotropic diffusion filter. The approach described in this paper overcomes the limitations of scalar and vector metrics and, for the first time, computes the exact amounts of detail blur occurring in anisotropic diffusion filtering. Many computer simulations are reported in the paper in order to show how detail blur depends upon the various parameter settings. Error maps showing the actual location of this kind of filtering error are also provided. This paper is organized as follows. Section II describes the theoretical evaluation of the PSBR, Section III focuses on many computer simulations, and, finally, Section IV reports conclusions.

\section{THEORETICAL EVALUATION OF THE PSBR}

In this section, we briefly review the PSBR approach. Then, we show how a formal expression for the detail blur (and then for the PSBR) can be directly obtained from anisotropic diffusion theory.

\section{A. Definition of PSBR}

Let us deal with digitized images having $\mathrm{L}$ gray levels (typically $\mathrm{L}=256$ ). Let $\mathrm{r}(\mathbf{n})$ be the pixel luminance at location $\mathbf{n}=\left[\mathrm{n}_{1}, \mathrm{n}_{2}\right]$ in the reference (noise-free) image. Let $x(\mathbf{n})=r(\mathbf{n})+\eta(\mathbf{n})$ be the noisy pixel at location $\mathbf{n}=\left[n_{1}, n_{2}\right]$, where 
$\eta(\mathbf{n})$ represents the noise amplitude. Finally, let $\mathrm{f}(\mathbf{n})$ be the pixel luminance at the same location in the filtered picture. Thus, we can express the PSNR as follows:

$$
P S N R=10 \log _{10} \frac{(L-1)^{2}}{\frac{1}{N} \sum_{\mathbf{n}} e^{2}(\mathbf{n})}
$$

where $e(\mathbf{n})=f(\mathbf{n})-r(\mathbf{n})$ is the filtering error and $\mathrm{N}$ is the total number of processed pixels. Now, let $B$ represent a measure of the detail blur in the overall image:

$$
B=\frac{1}{N} \sum_{\mathbf{n}} \hat{e}_{b}^{2}(\mathbf{n})
$$

where $\hat{e}_{b}(\mathbf{n})$ is an estimate of the actual error component $e_{b}(\mathbf{n})$ dealing with the detail blur. If $\mathrm{B} \neq 0$ (as commonly occurs during noise smoothing), we can split the PSNR into two components, namely peak signal-to-blur ratio (PSBR) and degradation caused by noise (D), as expressed by the following relationships:

\section{$P S N R=P S B R-D$}

$$
\begin{aligned}
& P S B R=10 \log _{10} \frac{(L-1)^{2}}{B} \\
& D=10 \log _{10} \frac{\frac{1}{N} \sum_{\mathbf{n}} e^{2}(\mathbf{n})}{B}
\end{aligned}
$$

The PSBR takes into account how good a filter is at preserving image details, whereas D defines the loss in image quality produced by unfiltered noise. If no noise is added to the input image, we have $\mathrm{D}=0$ and thus PSNR=PSBR, according to (3).

\section{B. Evaluating the true PSBR from anisotropic diffusion theory}

Let us consider the basic anisotropic diffusion algorithm dealing with a four-pixel neighborhood $\mathrm{W}=\left\{\mathrm{x}_{\mathrm{i}}(\mathbf{n})\right.$ : $\left.\mathrm{i}=1,2,3,4\right\}$ [42]:

$$
\begin{aligned}
& f^{(k+1)}(\mathbf{n})=f^{(k)}(\mathbf{n})+\lambda \sum_{i=1}^{4} c_{i}^{(k)}(\mathbf{n}) \Delta f_{i}^{(k)}(\mathbf{n}) \\
& \Delta f_{i}^{(k)}(\mathbf{n})=f_{i}^{(k)}(\mathbf{n})-f^{(k)}(\mathbf{n}) \\
& c_{i}^{(k)}(\mathbf{n})=g\left(\Delta f_{i}^{(k)}(\mathbf{n})\right)
\end{aligned}
$$

where $f^{(k)}(\mathbf{n})$ is the filter output at the $\mathrm{k}$-th iteration, $f_{i}^{(k)}(\mathbf{n})$ are the corresponding values of the neighboring pixels, $\Delta f_{i}^{(k)}(\mathbf{n})$ indicate nearest-neighbor differences, $c_{i}^{(k)}(\mathbf{n})$ are the conduction coefficients and $0 \leq \lambda \leq 0.25$ ensures numerical stability. Different functions can be adopted for the function $\mathrm{g}$, for example:

$$
g(\Delta)=\frac{1}{1+\left(\frac{|\Delta|}{K}\right)^{2}}
$$

where the constant $\mathrm{K}$ can be assigned. Remembering that $x(\mathbf{n})=r(\mathbf{n})+\eta(\mathbf{n})$, at the first iteration we have:

$$
\begin{aligned}
& f^{(1)}(\mathbf{n})=f^{(0)}(\mathbf{n})+\lambda \sum_{i=1}^{4} c_{i}^{(0)}(\mathbf{n}) \Delta f_{i}^{(0)}(\mathbf{n}) \\
& =r^{(0)}(\mathbf{n})+\eta^{(0)}(\mathbf{n})+\lambda \sum_{i=1}^{4} c_{i}^{(0)}(\mathbf{n})\left[\Delta r_{i}^{(0)}(\mathbf{n})+\Delta \eta_{i}^{(0)}(\mathbf{n})\right]
\end{aligned}
$$

where formally $r^{(0)}(\mathbf{n})=r(\mathbf{n}), \eta^{(0)}(\mathbf{n})=\eta(\mathbf{n}), f^{(0)}(\mathbf{n})=x(\mathbf{n})$, $\Delta f_{i}^{(0)}(\mathbf{n})=\Delta r_{i}^{(0)}(\mathbf{n})+\Delta \eta_{i}^{(0)}(\mathbf{n}), \Delta r_{i}^{(0)}(\mathbf{n})=r_{i}(\mathbf{n})-r(\mathbf{n})$, and $\Delta \eta_{i}^{(0)}(\mathbf{n})=\eta_{i}(\mathbf{n})-\eta(\mathbf{n})$. Now, we can rewrite (10) as follows:

$$
f^{(1)}(\mathbf{n})=r^{(1)}(\mathbf{n})+\eta^{(1)}(\mathbf{n})
$$

where:

$$
\begin{aligned}
& r^{(1)}(\mathbf{n})=r^{(0)}(\mathbf{n})+\lambda \sum_{i=1}^{4} c_{i}^{(0)}(\mathbf{n}) \Delta r_{i}^{(0)}(\mathbf{n}) \\
& \eta^{(1)}(\mathbf{n})=\eta^{(0)}(\mathbf{n})+\lambda \sum_{i=1}^{4} c_{i}^{(0)}(\mathbf{n}) \Delta \eta_{i}^{(0)}(\mathbf{n})
\end{aligned}
$$

The term $r^{(1)}(\mathbf{n})$ shows how the original noise-free value $r^{(0)}(\mathbf{n})=r(\mathbf{n})$ has been modified by the filtering (detail blur) whereas the term $\eta^{(1)}(\mathbf{n})$ represents the effect of the filtering on the noise (residual noise). It can be easily shown that the filter output at the iteration $\mathrm{k}+1$ is given by:

where:

$$
f^{(k+1)}(\mathbf{n})=r^{(k+1)}(\mathbf{n})+\eta^{(k+1)}(\mathbf{n})
$$

$$
\begin{aligned}
& r^{(k+1)}(\mathbf{n})=r^{(k)}(\mathbf{n})+\lambda \sum_{i=1}^{4} c_{i}^{(k)}(\mathbf{n}) \Delta r_{i}^{(k)}(\mathbf{n}) \\
& \eta^{(k+1)}(\mathbf{n})=\eta^{(k)}(\mathbf{n})+\lambda \sum_{i=1}^{4} c_{i}^{(k)}(\mathbf{n}) \Delta \eta_{i}^{(k)}(\mathbf{n})
\end{aligned}
$$

Thus, the filtering error $e^{(k+1)}(\mathbf{n})=f^{(k+1)}(\mathbf{n})-r(\mathbf{n})$ can be split into two components $e_{r}^{(k+1)}(\mathbf{n})$ and $e_{\eta}^{(k+1)}(\mathbf{n})$, as follows:

$$
\begin{aligned}
& e^{(k+1)}(\mathbf{n})=e_{r}^{(k+1)}(\mathbf{n})+e_{\eta}^{(k+1)}(\mathbf{n}) \\
& e_{r}^{(k+1)}(\mathbf{n})=r^{(k+1)}(\mathbf{n})-r(\mathbf{n})
\end{aligned}
$$




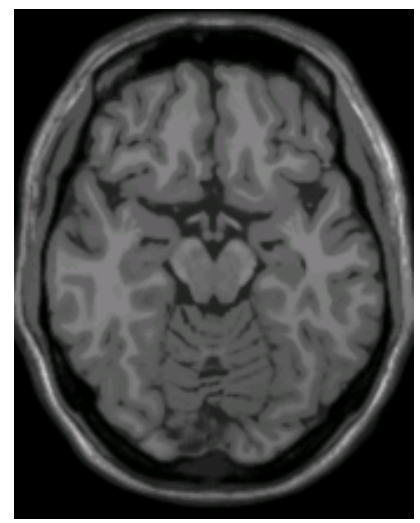

(a)

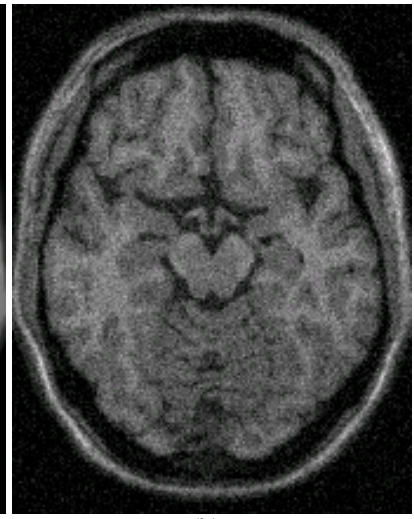

(b)

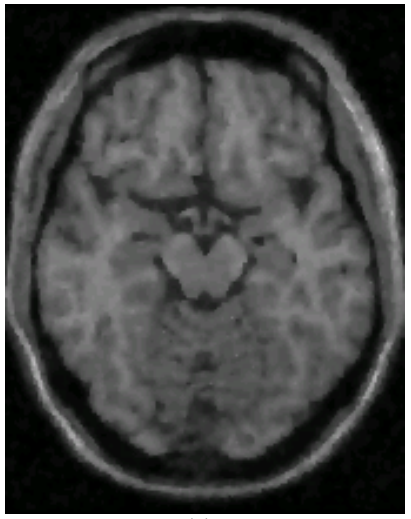

(c)

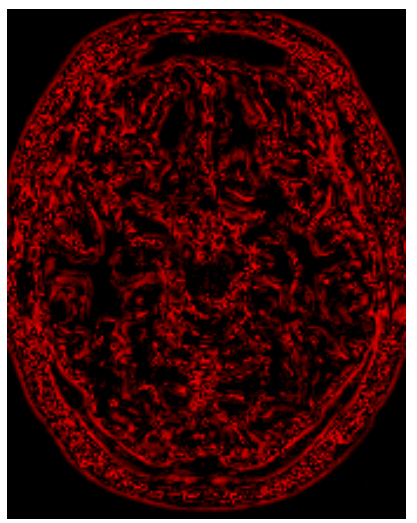

(d)

Fig.1 - Simulated BrainWeb data: (a) noise-free image, (b) image corrupted by Gaussian noise with $\sigma=15$, (c) result given by anisotropic filtering $(\lambda=0.125, \mathrm{~K}=19, \mathrm{PSNR}=31.10, \mathrm{PSBR}=36.60)$, (d) map of the corresponding errors due to filtering blur.

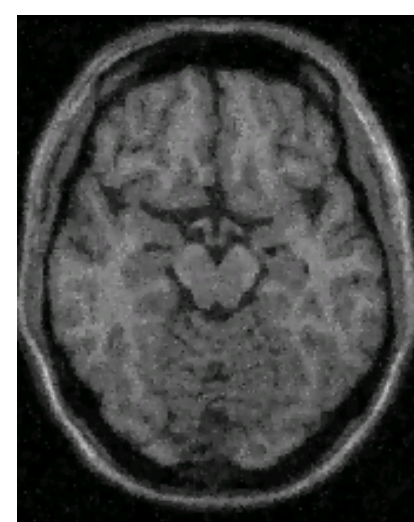

(a)

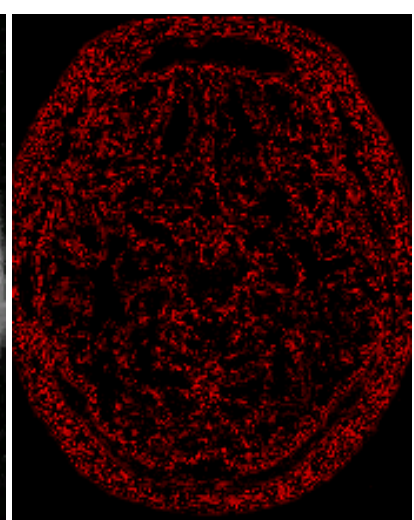

(b)

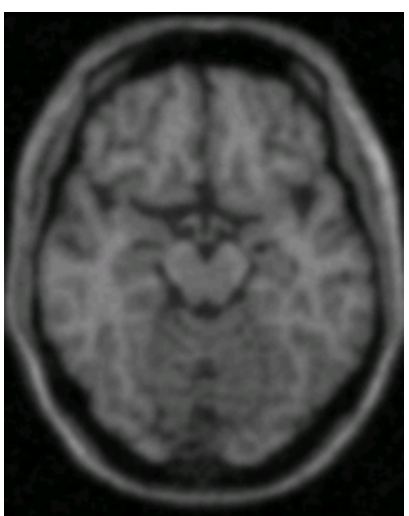

(c)

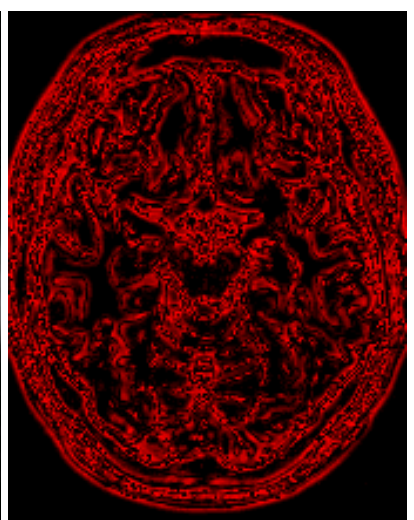

(d)

Fig. 2 - Example of filtered data having the same PSNR but different values of PSBR: (a) result given by choosing K=9 (PSNR=28.83, $\mathrm{PSBR}=36.60$, (b) map of the errors denoting filtering blur, (c) result given by $\mathrm{K}=86$ (PSNR=28.83, $\mathrm{PSBR}=30.35$ ), (d) errors denoting blur.

$$
e_{\eta}^{(k+1)}(\mathbf{n})=\eta^{(k+1)}(\mathbf{n})
$$

where $e_{r}^{(k+1)}(\mathbf{n})$ denotes the (signed) error component responsible for the generation of detail blur and $e_{\eta}^{(k+1)}(\mathbf{n})$ represents the error component dealing with the residual noise. Depending on the signs and amounts of $e_{r}^{(k+1)}(\mathbf{n})$ and $e_{\eta}^{(k+1)}(\mathbf{n})$, the actual detail blur $e_{b}^{(k+1)}(\mathbf{n})$ is evaluated as follows.

If $e_{r}^{(k+1)}(\mathbf{n})$ and $e_{\eta}^{(k+1)}(\mathbf{n})$ have the same signs, there is no compensation and then we have: $e_{b}^{(k+1)}(\mathbf{n})=e_{r}^{(k+1)}(\mathbf{n})$.

If $e_{r}^{(k+1)}(\mathbf{n})$ and $e_{\eta}^{(k+1)}(\mathbf{n})$ have different signs and $\left|e_{r}^{(k+1)}(\mathbf{n})\right| \geq\left|e_{\eta}^{(k+1)}(\mathbf{n})\right|$, detail blur prevails and then $e_{b}^{(k+1)}(\mathbf{n})=e^{(k+1)}(\mathbf{n})$. Finally, let us suppose that $e_{r}^{(k+1)}(\mathbf{n})$ and $e_{\eta}^{(k+1)}(\mathbf{n})$ have different signs and $\left|e_{r}^{(k+1)}(\mathbf{n})\right|<\left|e_{\eta}^{(k+1)}(\mathbf{n})\right|$. In this case $e_{b}^{(k+1)}(\mathbf{n})=0$.

Hence, the true PSBR (namely $\operatorname{PSBR}_{\mathrm{T}}$ ) yielded by the iteration $\mathrm{k}+1$ of the filter can be computed as follows:

$$
\operatorname{PSBR}_{T}^{(k+1)}=10 \log _{10} \frac{(L-1)^{2}}{\frac{1}{N} \sum_{\mathbf{n}}\left[e_{b}^{(k+1)}(\mathbf{n})\right]^{2}}
$$

Notice that, in general, $\left|e^{(k+1)}(\mathbf{n})\right| \neq\left|e_{r}^{(k+1)}(\mathbf{n})\right|+\left|e_{\eta}^{(k+1)}(\mathbf{n})\right|$, so we cannot always consider $e_{b}^{(k+1)}(\mathbf{n})$ as the absolute value of $e_{r}^{(k+1)}(\mathbf{n})$. As an example, if $e_{r}^{(k+1)}(\mathbf{n})=-3$ and $e_{\eta}^{(k+1)}(\mathbf{n})=5$, then we have $e^{(k+1)}(\mathbf{n})=2$ and $e_{b}^{(k+1)}(\mathbf{n})=0$. Conversely, if $e_{r}^{(k+1)}(\mathbf{n})=3$ and $e_{\eta}^{(k+1)}(\mathbf{n})=5$, we have $e^{(k+1)}(\mathbf{n})=8 \quad$ and $e_{b}^{(k+1)}(\mathbf{n})=e_{r}^{(k+1)}(\mathbf{n})=3$. (An example dealing with actual data will be provided in the next Section).

\section{RESULTS OF COMPUTER SIMULATIONS}

We shall consider in this section some application examples dealing with simulated BrainWeb data [43-46]. 


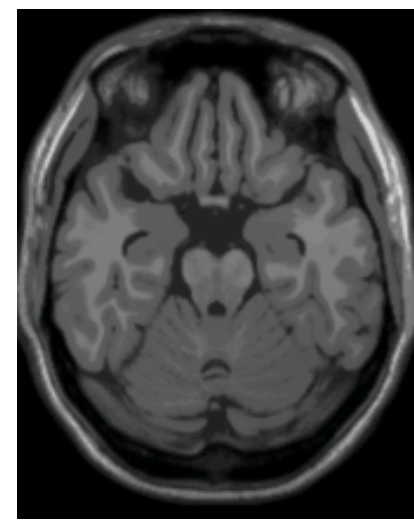

(a)

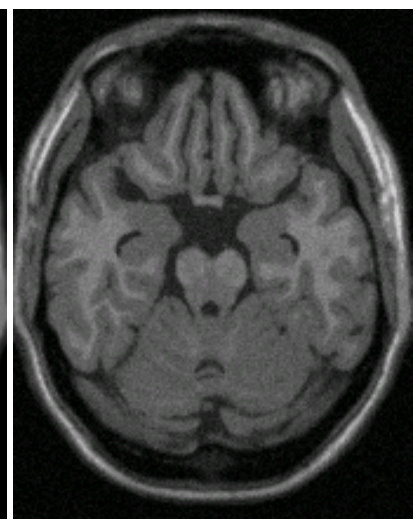

(b)

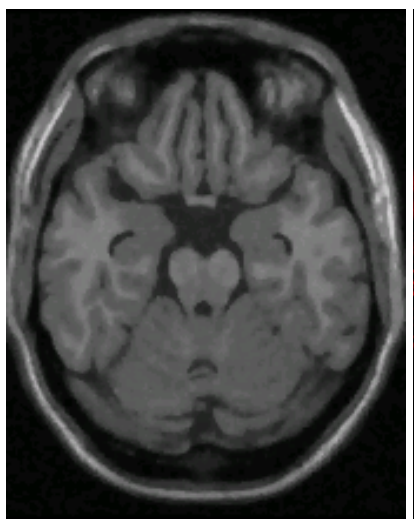

(c)

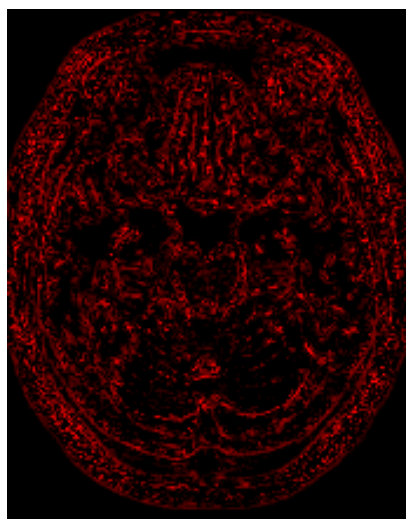

(d)

Fig.3 - Simulated BrainWeb data corrupted by Rician noise: (a) noise-free image, (b) noisy image, (c) result given by anisotropic diffusion filtering after five iterations $(\lambda=0.125, \mathrm{~K}=12)$, (d) map of the error components addressing detail blur.

In the first experiment we considered an example of anisotropic diffusion filtering where the main parameter $\mathrm{K}$ is chosen in order to achieve the maximum PSNR. The noisefree data are graphically represented in Fig.1a. The image in Fig. $1 \mathrm{~b}$ has been generated by adding zero-mean Gaussian noise with standard deviation $\sigma=15$. The result obtained after ten iterations of the anisotropic diffusion filter $(\lambda=0.125, K=19)$ is shown in Fig1c. From visual inspection we clearly see that the noise removal has been obtained at the price of some filtering blur. Now, we can achieve a quantitative evaluation of this effect. Indeed, according to (20), we have: $P S N R=31.10$ and $\operatorname{PSBR}_{T}=36.60$. The map in Fig.1d shows location and magnitude (amplified by 20) of the absolute values of the actual errors $\mathrm{e}_{b}(\mathbf{n})$ caused by filtering blur (red pixels). In the second experiment we considered the case of two filtered pictures having the same value of PSNR but different amounts of filtering distortion. The noisy input data are the same as in the previous experiment. Fig.2a shows the result given by $\mathrm{K}=9$ (PSNR=28.83, $\left.\mathrm{PSBR}_{\mathrm{T}}=36.60\right)$, whereas Fig.2c shows the result given by $\mathrm{K}=86\left(\mathrm{PSNR}=28.83, \mathrm{PSBR}_{\mathrm{T}}=30.35\right)$. The maps of the errors caused by filtering blur are reported in Fig2b and $2 \mathrm{~d}$, respectively. Clearly, the detail preservation is worse for the picture in Fig.2c with respect to the image in Fig.2a. Unlike all other metrics, the $\mathrm{PSBR}_{\mathrm{T}}$ can exactly evaluate how much these data are blurred. A further experiment dealing with simulated BrainWeb data corrupted by Rician noise is considered in Fig.3. The noise-free picture is shown in Fig.3a while the corrupted data are depicted in Fig.3b. The result of the anisotropic diffusion algorithm (five iterations) yielding the best PSNR is reported in Fig.3c (PSNR=33.50, PSBR $\left._{\mathrm{T}}=40.74\right)$. The map of corresponding errors due to filtering blur is reported in Fig.3d. The values of PSNR and $\mathrm{PSBR}_{\mathrm{T}}$ that are obtained when the main parameter $K$ ranges from 2 to 40 are graphically depicted in Fig.4 for five and ten iterations of the filter. Finally, the results of some tests dealing with the well-known Shepp-Logan phantom image are depicted in Figs.5 and 6. The correct behavior of the $\mathrm{PSBR}_{\mathrm{T}}$ is apparent in Fig.6a (five iterations) and Fig.6b (ten iterations). As the value of the main parameter $\mathrm{K}$ becomes larger (and so the filtering blur), the value of $\mathrm{PSBR}_{\mathrm{T}}$ decreases, as it should be. For a comparison, we considered the Quality Index based on Local Variance (QILV) [47]. This is an interesting full-reference quality index that, like other techniques [35], measures the structural similarity between the filtered and the original noise-free images. The QILV is more sensitive to detail blur than to the amount of noise: the larger the blur the smaller its value should be. If we observe the data in Fig.6, however, we see that the QILV wrongly increases for growing values of $\mathrm{K}: 5 \leq \mathrm{K} \leq 20$ (Fig,6a) and $5 \leq \mathrm{K} \leq 10$ (Fig,6b). Thus, similar values of QILV can be obtained for images having different amounts of detail blur. This never occurs using the $\mathrm{PSBR}_{\mathrm{T}}$. As an example (Fig.6a), for $\mathrm{K}=5$ we have QILV=0.827 and $\mathrm{PSBR}_{\mathrm{T}}=54.869 \mathrm{~dB}$, whereas for $\mathrm{K}=52.6$ we have $\mathrm{QILV}=0.827$ and $\mathrm{PSBR}_{\mathrm{T}}=37.034 \mathrm{~dB}$.

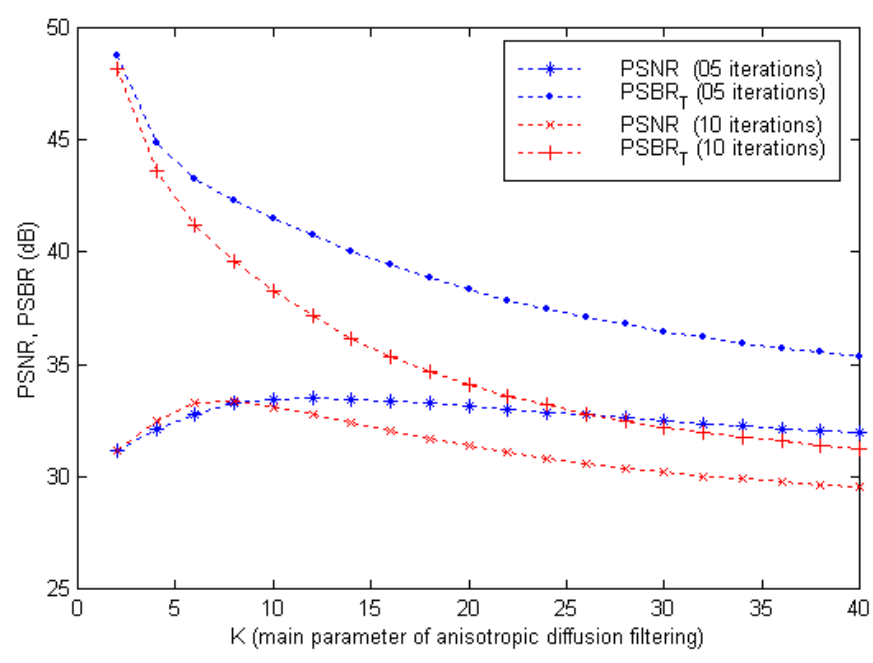

Fig.4 - Simulated BrainWeb data corrupted by Rician noise: PSNR and $\mathrm{PSBR}_{\mathrm{T}}$ evaluations for the results given by anisotropic diffusion filtering $(\lambda=0.125, \quad 2 \leq \mathrm{K} \leq 40)$. 


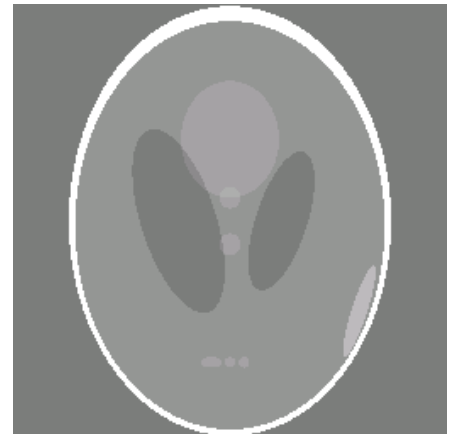

(a)

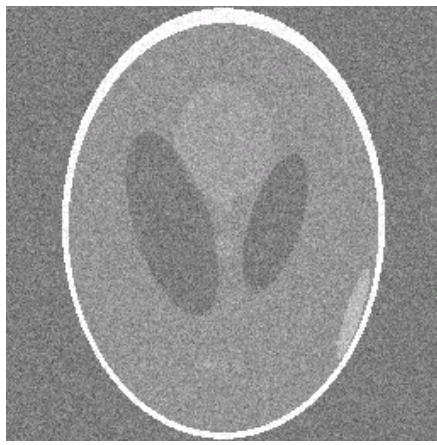

(b)

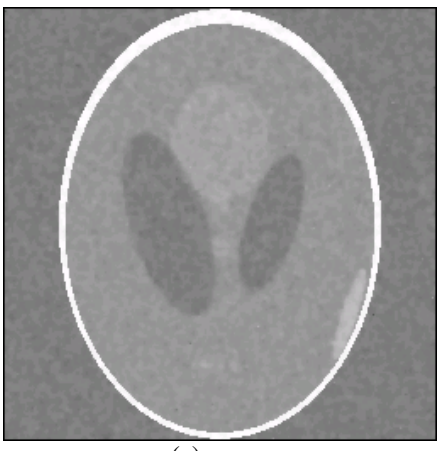

(c)

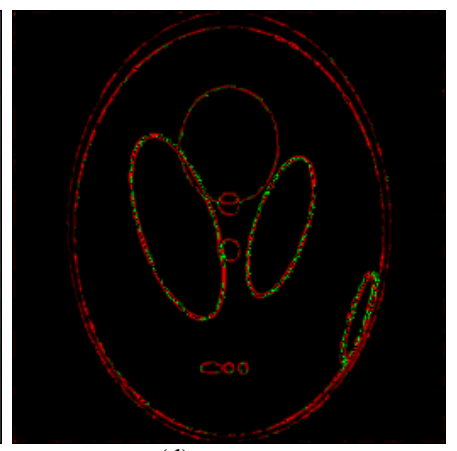

(d)

Fig. 5 - (a) Shepp-Logan phantom image, (b) image corrupted by Gaussian noise with $\sigma=15$, (c) result given by anisotropic diffusion filtering after ten iterations $(\lambda=0.125, \mathrm{~K}=20)$, (d) map of the error components addressing detail blur $\left(\left|e_{b}(\mathbf{n})\right|=\left|e_{r}(\mathbf{n})\right| \rightarrow \operatorname{red},\left|e_{b}(\mathbf{n})\right| \neq\left|e_{r}(\mathbf{n})\right| \rightarrow \operatorname{green}\right)$.

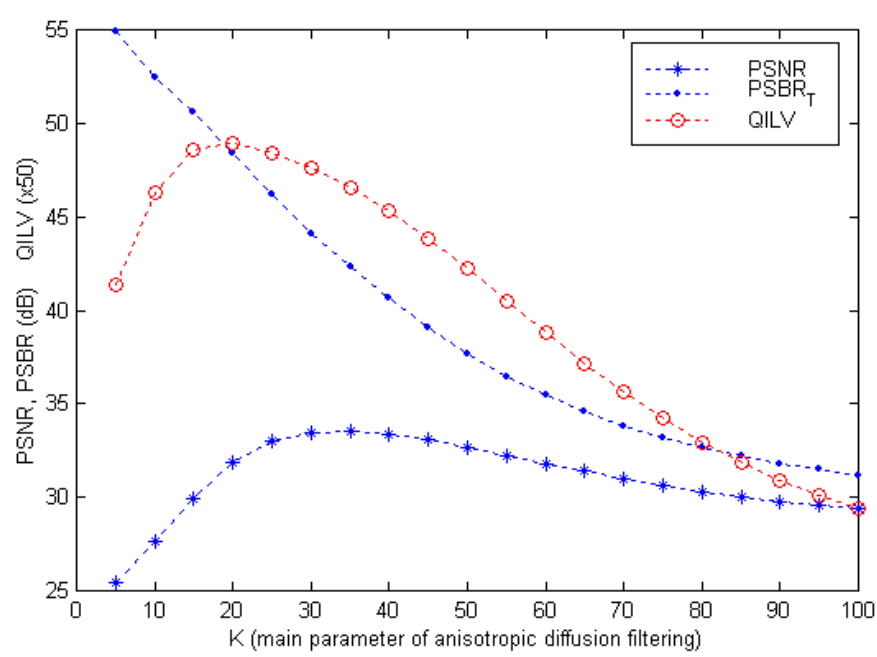

(a)

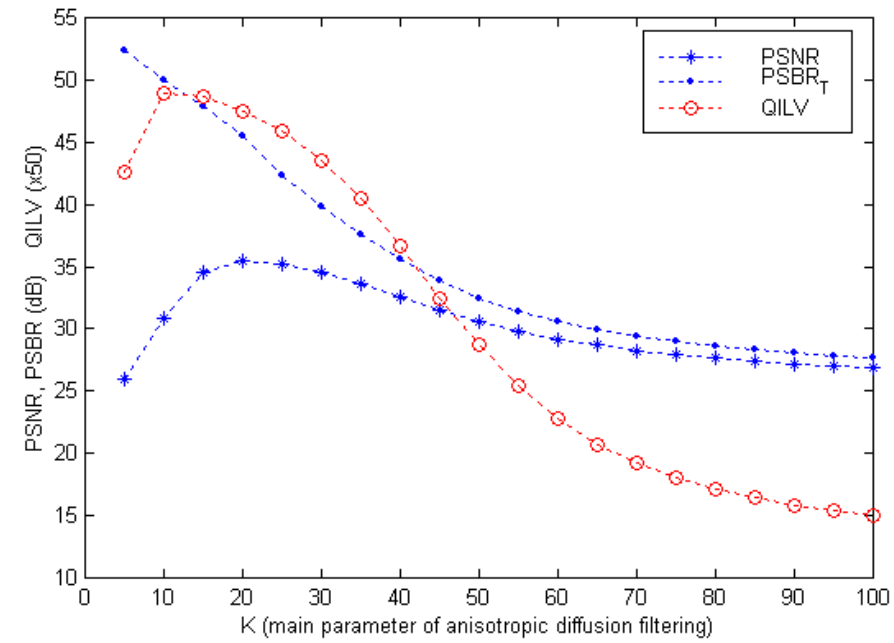

(b)

Fig.6 - Shepp-Logan phantom image corrupted by Gaussian noise: PSNR, PSBR $\mathrm{T}$ and QILV $(\times 50)$ evaluations for the results given by anisotropic diffusion filtering $(\lambda=0.125,5 \leq \mathrm{K} \leq 100)$ adopting five iterations (a) and ten iterations (b).

\section{CONCLUSIONS}

In this paper we have presented a novel method for investigating the accuracy of anisotropic diffusion filters. Instead of resorting to existing metrics that try to estimate the filtering blur, we have shown how, under the hypothesis of additive noise, the exact amount of detail preservation yielded by an anisotropic diffusion filter can be formally derived from the filter theory. Results of computer simulations have shown that the true value of PSBR can be easily computed and used in conjunction with the well-known PSNR in order to fully characterize the filtering behavior. The method lies in the framework of measurement science, where the concept of error as the difference between the result and the true value plays a very relevant role. Indeed, the method can yield a quantitative evaluation of filtering distortion without the possible inaccuracies of the commonly adopted full-reference metrics. It can also provide error maps showing amount and location of this kind of filtering errors.

\section{REFERENCES}

[1] P. Milanfar, "A tour of modern image filtering: New insights and methods, both practical and theoretical," IEEE Signal Process. Mag., vol.30, n.1, 2012, pp.106-128.

[2] L. Shao, R. Yan, X. Li, and Y. Liu, "From heuristic optimisation to dictionary learning: a review and comprehensive comparison of image denoising algorithms," IEEE Trans. Cybern., vol.44, n.7, 2014, pp.1001-1013.

[3] P. Perona and J. Malik, "Scale-space and edge detection using anisotropic diffusion," IEEE Trans. Pattern Anal. Mach. Intell., vol.12, no. 7, 1990, pp. 629-639.

[4] G. Gerig, O. Kübler, R. Kikinis, and F. Jolesz, "Nonlinear anisotropic filtering of MRI data," IEEE Trans. Med. Imag., vol. 11, no. 6, 1992, pp.221-232.

[5] J. Weickert, B. M. T. H. Romeny, and M. Viergever, "Efficient and reliable schemes for nonlinear diffusion filtering," IEEE Trans. Image Process., vol.7, n.3, 1998, pp.398-410.

[6] D. Barash, "Fundamental relationship between bilateral filtering, adaptive smoothing, and the nonlinear diffusion equation," IEEE Trans. Pattern Anal. Mach. Intell., vol.24, n.6, 2002, pp.844-847.

[7] G. Ramos-Llordén, G. Vegas-Sánchez-Ferrero, M. Martin-Fernandez, C. Alberola-López and S. Aja-Fernández, "Anisotropic diffusion filter with 
memory based on speckle statistics for ultrasound images", IEEE Trans. on Image Processing, Vol.24, n.1, 2015, pp.345-358.

[8] V. Ostojic, D. Starcevic and V. Petrovic, "Recursive anisotropic diffusion denoising", Electronics Letters, vol.52 n.17, 2016, pp. 14491451 .

[9] D. Mishra , S. Chaudhury, M. Sarkar, A. Singh Soin, and V. Sharma,"Edge probability and pixel relativity-based speckle reducing anisotropic diffusion", IEEE Trans. on Image Processing, vol.27, n.2, 2018, pp.649-664.

[10] A. Buades, B. Coll, and J.-M. Morel, "A review of image denoising algorithms, with a new one," Multiscale Model. Simul., vol.4, n.2, 2005, pp. $490-530$.

[11] M. Mahmoudi and G. Sapiro, "Fast image and video denoising via nonlocal means of similar neighborhoods", IEEE Signal Process. Lett., Vol.12, n.1, 2005, pp.839-842.

[12] P. Coupé, P. Yger, S. Prima, P. Hellier, C. Kervrann, and C. Barillot, "An optimized blockwise nonlocal means denoising filter for 3-D magnetic resonance images”, IEEE Trans. on Medical Imaging, vol.27, n.4, 2008, pp.425-441.

[13] V. May, Y. Keller, N. Sharon, and Y. Shkolnisky, "An algorithm for improving non-local means operators via low-rank approximation", IEEE Trans. Image Process, vol.25, n.3, 2016, pp.1340-1353.

[14] V. Fedorov and C. Ballester, "Affine non-local means image denoising", IEEE Trans. Image Process, vol. 26, n.5, 2017, pp.2137-2148.

[15] I. D. Dinov, M. S. Mega, P. M. Thompson, R. P. Woods, De Witt L. Sumners, E. L. Sowell, and A. W. Toga, "Quantitative comparison and analysis of brain image registration using frequency-adaptive wavelet shrinkage", IEEE Trans. on Information Tech. in Biomedicine, vol.6, n.1, 2002, pp.73-85

[16] A. Pizurica, W. Philips, I. Lemahieu and M. Acheroy, "A Versatile Wavelet Domain Noise Filtration Technique for Medical Imaging", IEEE Trans. On Medical Imaging, vol.22, n.3, pp.2003, 323-331.

[17] A. Meije Wink and J. B. T. M. Roerdink, "Denoising Functional MR Images: A Comparison of Wavelet Denoising and Gaussian Smoothing", IEEE Trans. on Medical Imaging, vol.23, n.3, 2004, pp.374-387.

[18] A. Mencattini, M. Salmeri, R. Lojacono, M. Frigerio, and F. Caselli,"Mammographic images enhancement and denoising for breast cancer detection using dyadic wavelet processing transform," IEEE Trans. Instrum. Meas., vol.57, n.7, 2008, pp. 1422-1430.

[19] G.Andria, F Attivissimo, G. Cavone, A.M.L. Lanzolla, "Selection of wavelet functions and thresholding parameteres in ultrasound image denoising", in Proc. MeMeA 2013, Gatineau, QC, Canada, May 4-5, 2013, pp. 49-52.

[20] A. Dineva, A. R. Várkonyi-Kóczy, J. K. Tar, "Fuzzy expert system for automatic wavelet shrinkage procedure selection for noise suppression", Proc. INES 2014, IEEE 18th International Conference on Intelligent Engineering Systems, July 3-5, 2014, Tihany, Hungary.

[21] A. Ouahabi, "A review of wavelet denoising in medical imaging", 2013 $8^{\text {th }}$ International Workshop on Systems, Signal Processing and their Applications (WoSSPA), Algiers, 2013, pp. 19-26.

[22] S. Aja-Fernández and C. Alberola-López, "On the estimation of the coefficient of variation for anisotropic. diffusion speckle filtering", IEEE Trans. on Image Processing, vol.15, n.9, 2006, pp.2694-2701.

[23] G. Andria, F. Attivissimo, A. M. L. Lanzolla and M. Savino, "A suitable threshold for speckle reduction in ultrasound images", IEEE Trans. on Instrum. Meas., vol.62, n.8, 2013, pp.2270-2279.

[24] D. Gupta, R. Shyam Anand, B. Tyagi, "Speckle filtering of ultrasound images using a modified non-linear diffusion model in non-subsampled shearlet domain", IET Image Process., vol.9, n.2, 2015, pp. 107-117.

[25] M. Rafati, M. Arabfard, M. R. R. Zadeh, M. Maghsoudloo, "Assessment of noise reduction in ultrasound images of common carotid and brachial arteries", IET Comput. Vis., vol.10, n.1, 2016, pp.1-8.

[26] F. Attivissimo, G. Cavone, A. M. L. Lanzolla, and M. Spadavecchia, "A technique to improve the image quality in computer tomography", IEEE Transactions on Instrumentation and Measurement, vol.59, n.5, 2010, pp.1251-1257.
[27] A. M. Mendrik, E.-J. Vonken, A. Rutten, M. A. Viergever and B. van Ginneken, "Noise reduction in computed tomography scans using 3-D anisotropic hybrid diffusion with continuous switch", IEEE Trans. on Medical Imaging, vol.28, n.10, 2009, 1585-1594.

[28] S. P. Awate and R. T. Whitaker, "Feature-preserving MRI denoising: a nonparametric empirical Bayes approach", IEEE Trans. on Medical Imaging, vol.26, n.9, 2007, pp.1242-1255.

[29] K. Krissian and S. Aja-Fernández, "Noise-driven anisotropic diffusion filtering of MRI", IEEE Trans. on Image Processing, vol.18, n.10, 2009 pp.2265-2274.

[30] L. He and I. R. Greenshields, "A nonlocal maximum likelihood estimation method for Rician noise reduction in MR images", IEEE Trans. on Medical Imaging, vol.28, n..2, 2009, pp.165-172.

[31] B. Kang, O. Choi, J.D. Kim and D. Hwang, "Noise reduction in magnetic resonance images using adaptive non-local means filtering", Elecronics Letters, vol.49, n.5, 2013.

[32] S. Aja-Fernandez C. Alberola-Lopez C. F. Westin, "Noise and signal estimation in magnitude MRI and Rician distributed images: A LMMSE approach", IEEE Trans. Image Processing vol.17, n.8, Aug. 2008, pp.1383-1398.

[33] D. L. Carnì, D. Grimaldi, and F. Lamonaca, "Preprocessing correction for micronucleus image detection affected by contemporaneous alterations", IEEE Transactions on Instrumentation and Measurement, vol.56, n.4, 2007, pp.1202-1211.

[34] F. Adamo, F. Attivissimo, A. Di Nisio, and M. Spadavecchia, "An automatic document processing system for medical data extraction", Measurement, Elsevier, vol.61, 2015, pp.88-89.

[35] Z. Wang, A. C. Bovik, H. R. Sheikh, and E. P. Simoncelli, "Image quality assessment: from error visibility to structural similarity", IEEE Transactions on Image Processing, vol.13, n.4, 2004, pp.600-612.612.

[36] S. Winkler and P. Mohandas, "The evolution of video quality measurement: from PSNR to hybrid metrics", IEEE Transactions on Broadcasting, Vol.54, n.3, 2008, pp.660-668.

[37] F. Russo, "New method for performance evaluation of grayscale image denoising filters", IEEE Signal Processing Letters, vol.17, n.5, 2010, pp.417-420.

[38] F. Russo, " New method for measuring the detail preservation of noise removal techniques in digital images", WSEAS Transactions on Signal Processing, Vol. 11, n.38, 2015, pp. 317-327.

[39] A. De Angelis, A. Moschitta, F. Russo and P. Carbone, "A vector approach for image quality assessment and some metrological considerations," IEEE Transactions on Instrumentation and Measurement, vol.58, n.1, 2009, pp. 14-25.

[40] F. Russo, "Validation of denoising algorithms for medical imaging", in "Advances in Biomedical Sensing, Measurements, Instrumentation and Systems", S.C. Mukhopadhyay and A. Lay-Ekuakille (Eds.), SpringerVerlag, 2010, pp. 93-105.

[41] F. Russo, "On the accuracy of vector metrics for quality assessment in image filtering", in Proc. $20^{\text {th }} 2014$ IMEKO TC4 International Symposium, Benevento, Italy, September 15-17, 2014, pp.863-868.

[42] I. Pitas, Digital Image Processing Algorithms ans Applications, John Wiley \& Sons Inc. 2000.

[43] http://www.bic.mni.mcgill.ca/brainweb/

[44] C.A. Cocosco, V. Kollokian, R.K.-S. Kwan and A.C. Evans, "BrainWeb: online interface to a 3D MRI simulated brain database", NeuroImage, vol.5, no.4, 1997, part 2/4, S425.

[45] R.K.-S. Kwan, A.C. Evans and G.B. Pike, "MRI simulation-based evaluation of image-processing and classification methods", IEEE Transactions on Medical Imaging., vol.18, n.11, 1999, pp.1085-97.

[46] R.K.-S. Kwan, A.C. Evans and G.B. Pike, “An extensible MRI simulator for post-processing evaluation", Visualization in Biomedical Computing (VBC'96). Lecture Notes in Computer Science, vol. 1131, 1996, Springer-Verlag.

[47] S. Aja-Fernández, R. San Jose Estepar, C. Alberola- López and C.-F. Westin, "Image Quality Assessment based on Local Variance", Proc. EMBS Annual International Conference New York City, USA, Aug 30Sept 3, 2006, pp.4815-4818. 